\title{
Approach to the glass transition studied by higher order correlation functions
}

\author{
N Lačević and S C Glotzer \\ Departments of Chemical Engineering and Materials Science and Engineering, \\ University of Michigan, Ann Arbor, MI 48109, USA \\ E-mail: sglotzer@umich.edu
}

Received 11 June 2003

Published 23 July 2003

Online at stacks.iop.org/JPhysCM/15/S2437

\begin{abstract}
We present a theoretical framework based on a higher order density correlation function, analogous to that used to investigate spin glasses, to describe dynamical heterogeneities in simulated glass-forming liquids. These higher order correlation functions are a four-point, time-dependent density correlation function $g_{4}(r, t)$ and a corresponding 'structure factor' $S_{4}(q, t)$ which measure the spatial correlations between the local liquid density at two points in space, each at two different times. $g_{4}(r, t)$ and $S_{4}(q, t)$ were extensively studied via molecular dynamics simulations of a binary Lennard-Jones mixture approaching the mode coupling temperature from above in Franz et al (1999 Phil. Mag. B 79 1827), Donati et al (2002 J. Non-Cryst. Solids 307 215), Glotzer et al (2000 J. Chem. Phys. 112 509), Lačević et al (2002 Phys. Rev. E 66 030101), Lačević et al (2003 J. Chem. Phys. submitted) and Lačević (2003 Dissertation The Johns Hopkins University). Here, we examine the contribution to $g_{4}(r, t), S_{4}(q, t)$ and the corresponding dynamical correlation length, as well as the corresponding order parameter $Q(t)$ and generalized susceptibility $\chi_{4}(t)$, from localized particles. We show that the dynamical correlation length $\xi_{4}^{\mathrm{SS}}(t)$ of localized particles has a maximum as a function of time $t$, and the value of the maximum of $\xi_{4}^{\text {SS }}(t)$ increases steadily in the temperature range approaching the mode coupling temperature from above.
\end{abstract}

\section{Introduction}

One of the most challenging problems in condensed matter physics is understanding the dynamics and thermodynamics of glass formation [6]. On cooling from high temperature, liquids may crystallize at $T_{\mathrm{m}}$, or if a liquid is cooled so that crystallization is avoided, it may become supercooled. As a supercooled liquid is further cooled, the particles move more and more slowly, and their motion is slowed down so drastically that, at some low $T$, particles will not be able to rearrange. This rearrangement is necessary for a liquid to find its equilibrium, 
and at this temperature particles in a liquid appear to be 'frozen' or 'jammed', at least for the timescale of an experiment. At $T_{\mathrm{g}}$ the supercooled liquid is no longer in equilibrium. This is called a glass transition, and the temperature at which this occurs is called the glass transition temperature $T_{\mathrm{g}}$. An extensive treatment of the dynamics and thermodynamics of supercooled liquids can be found in the textbooks by Debenedetti [7], Harrison [8] and Knight [9]. There are many conference proceedings [10-15] on experimental developments, theoretical advances and the role of computer simulation in the search for a universal theory of supercooled liquids and the glass transition.

We are still left with unanswered questions about the dramatic slowing down in the molecular motion as the temperature is lowered to $T_{\mathrm{g}}$, which would not be surprising alone, but this process is accompanied with no significant change in the long-range structure. How is it possible that dynamics changes so dramatically while the structure, as measured by traditional static two-point correlation functions, remains almost unchanged, and what type of transition is the glass transition are some of the crucial questions in the field of glass transition research. To be able to answer those questions, we must fully understand the dynamics of particles at the microscopic level, i.e. search for the patterns in particle motion and relate them to a mechanism for slowing down.

The prominent features of supercooled liquids are the non-Arrhenius temperature dependence of the viscosity and structural relaxation time [16], non-exponential character of the structural relaxation time [17], absence of changes in static structural two point quantities [1821] and decoupling of the transport coefficients [22-25]. In particular, the non-exponential character of the relaxation of density correlation functions and decoupling of the transport coefficients can be rationalized with the existence of spatially heterogeneous dynamics (SHD) or 'dynamical heterogeneity' now well established in experiment and computer simulation [2630]. Therefore, understanding of the origin of dynamical heterogeneity is directly related to understanding the origin of the glass transition. We refer to a system as dynamically heterogeneous if it is possible to select a dynamically distinguishable subset of particles by experiment or computer simulation [31]. From the theoretical point of view, the question 'what is dynamical heterogeneity?' still has different answers depending on the theoretical framework used to describe it. Recently, a new theoretical approach to the problem of cooperativity demonstrated how SHD can arise in simple systems with cooperative dynamics. This theory predicts a growing correlation length on decreasing $T$ [32]. Here we use a four-point, time-dependent, density correlation function formalism to select a dynamically distinguishable subset of particles [1-5]. In particular, we investigate the properties of those particles deemed to be localized.

\section{Method and model}

The simulation method we use to generate data for our analyses is molecular dynamics (MD). This is a widely used method in the investigation of supercooled liquids and glasses that provides static and dynamic properties for a collection of particles. The code we use in our simulations is LAMMPS ${ }^{1}$, a parallel MD code based on a spatial decomposition parallel technique.

We study a 50/50 binary mixture of particle types 'A' and 'B' that interact via the LennardJones potential

$$
V_{\alpha \beta}(r)=4 \epsilon_{\alpha \beta}\left[\left(\frac{\sigma_{\alpha \beta}}{r}\right)^{12}-\left(\frac{\sigma_{\alpha \beta}}{r}\right)^{6}\right] .
$$

1 Steve Plimpton, Sandia National Labs www.cs.sandia.gov/ $\sim$ sjplimp. 
This system has been previously studied by Wahnstrom [33] and Schrøder [34]. Following these authors, we use length parameters $\sigma_{A A}=1, \sigma_{B B}=5 / 6$ and $\sigma_{A B}=\left(\sigma_{A A}+\sigma_{B B}\right) / 2$, and energy parameters $\epsilon_{A A}=\epsilon_{B B}=\epsilon_{A B}=1$. The masses of the particles are chosen to be $m_{A}=2$ and $m_{B}=1$. We shift the potential and truncate it so it vanishes at $r=2.5 \sigma_{A B}$.

We simulate a system of $N=8000$ particles using periodic boundary conditions in a cubic box of length $L=18.334$ in units of $\sigma_{A A}$, which yields a density of $\rho=N / L^{3}=1.296$ for all state points. We report time in units of $\tau=\left(m_{B} \sigma_{A A}^{2} / 48 \epsilon_{A A}\right)^{1 / 2}$, length in units of $\sigma_{A A}$ and temperature, $T$, in units of $\epsilon_{A A} / k_{\mathrm{B}}$, where $k_{\mathrm{B}}$ is Boltzmann's constant. We simulate eight state points at temperatures ranging from $T=2.0$ to 0.59 , following a path similar to that followed in [2,34-36]. The simulations are performed in the $N V E$ ensemble. We estimate the mode coupling temperature $T_{\mathrm{MCT}}=0.57 \pm 0.01$ (the glass transition temperature $T_{\mathrm{g}}$ is typically in the range $0.6 T_{\mathrm{MCT}}<T_{\mathrm{g}}<0.9 T_{\mathrm{MCT}}$ [37]) and the Kauzmann temperature $T_{0}$, which can be considered a lower bound for the glass transition temperature $T_{g}, T_{0}=0.48 \pm 0.02$. How we estimate these temperatures and other simulation details can be found in $[4,5]$.

\section{Background}

In this section, we briefly review the theoretical framework of the four-point, spatiotemporal density correlation function and corresponding structure factor. Detailed derivation of these quantities can be found in [1-5].

We consider a liquid of $N$ particles occupying a volume $V$ with density $\rho(r, t)=$ $\sum \delta\left(\boldsymbol{r}-\boldsymbol{r}_{i}(t)\right)$, and investigate a quantity

$$
Q(t)=\int \mathrm{d} \boldsymbol{r}_{1} \mathrm{~d} \boldsymbol{r}_{2} \rho\left(\boldsymbol{r}_{1}, 0\right) \rho\left(\boldsymbol{r}_{2}, t\right) w\left(\left|\boldsymbol{r}_{1}-\boldsymbol{r}_{2}\right|\right)=\sum_{i=1}^{N} \sum_{j=1}^{N} w\left(\left|\boldsymbol{r}_{i}(0)-\boldsymbol{r}_{j}(t)\right|\right),
$$

which measures the number of particles that, in time $t$, either remain within a distance $a$ of their original position, or are replaced by another particle ('overlapping particles'). The reason for introducing an 'overlap' function $w$ is to eliminate weakly correlated vibrational motion of the particles (for more details see e.g. [4]). The fluctuation in $Q(t)$ may be defined as

$$
\chi_{4}(t)=\frac{\beta V}{N^{2}}\left[\left\langle Q(t)^{2}\right\rangle-\langle Q(t)\rangle^{2}\right]
$$

Expressing $\chi_{4}(t)$ in terms of the four-point correlation function $G_{4}\left(\boldsymbol{r}_{1}, \boldsymbol{r}_{2}, \boldsymbol{r}_{3}, \boldsymbol{r}_{4}, t\right)$, we obtain

$$
\chi_{4}(t)=\frac{\beta V}{N^{2}} \int \mathrm{d} \boldsymbol{r}_{1} \mathrm{~d} \boldsymbol{r}_{2} \mathrm{~d} \boldsymbol{r}_{3} \mathrm{~d} \boldsymbol{r}_{4} G_{4}\left(\boldsymbol{r}_{1}, \boldsymbol{r}_{2}, \boldsymbol{r}_{3}, \boldsymbol{r}_{4}, t\right)
$$

where

$$
\begin{gathered}
G_{4}\left(\boldsymbol{r}_{1}, \boldsymbol{r}_{2}, \boldsymbol{r}_{3}, \boldsymbol{r}_{4}, t\right)=\left\langle\rho\left(\boldsymbol{r}_{1}, 0\right) \rho\left(\boldsymbol{r}_{2}, t\right) w\left(\left|\boldsymbol{r}_{1}-\boldsymbol{r}_{2}\right|\right) \rho\left(\boldsymbol{r}_{3}, 0\right) \rho\left(\boldsymbol{r}_{4}, t\right) w\left(\left|\boldsymbol{r}_{3}-\boldsymbol{r}_{4}\right|\right)\right\rangle \\
-\left\langle\rho\left(\boldsymbol{r}_{1}, 0\right) \rho\left(\boldsymbol{r}_{2}, t\right) w\left(\left|\boldsymbol{r}_{1}-\boldsymbol{r}_{2}\right|\right)\right\rangle\left\langle\rho\left(\boldsymbol{r}_{3}, 0\right) \rho\left(\boldsymbol{r}_{4}, t\right) w\left(\left|\boldsymbol{r}_{3}-\boldsymbol{r}_{4}\right|\right)\right\rangle .
\end{gathered}
$$

Note that in the case of both the mean-field, p-spin model and a liquid in the hypernetted chain approximation [38-41], the time dependence of $\chi_{4}(t)$ was calculated numerically from an analytic expression in [1]. Those calculations provide the first analytical prediction of the growth of a generalized dynamical susceptibility and, by inference, a corresponding dynamical correlation length $\xi_{4}(t)$ in a model glass-forming system.

We wish to radially average the four-point correlation function in equation (5) to obtain a function $g_{4}(r, t)$ that depends only on the magnitude $r$ of the distance between two particles at time $t=0$. We start from the requirement that

$$
\chi_{4}(t)=\beta \int \mathrm{d} \boldsymbol{r} g_{4}(\boldsymbol{r}, t),
$$


and obtain

$g_{4}(\boldsymbol{r}, t)=\frac{1}{N \rho}\left\langle\sum_{i j k l} \delta\left(\boldsymbol{r}-\boldsymbol{r}_{k}(0)+\boldsymbol{r}_{i}(0)\right) w\left(\left|\boldsymbol{r}_{i}(0)-\boldsymbol{r}_{j}(t)\right|\right) w\left(\left|\boldsymbol{r}_{k}(0)-\boldsymbol{r}_{l}(t)\right|\right)\right\rangle-\left\langle\frac{Q(t)}{N}\right\rangle^{2}$.

Assuming an isotropic, homogeneous system, $g_{4}(\boldsymbol{r}, t)$ is a function of $r=|\boldsymbol{r}|$ (i.e. $g_{4}(r, t)$ ). Details of the above derivation can be found in $[4,5] . g_{4}(r, t)$ describes spatial correlations between overlapping particles separated by a distance $r$ at the initial time (using information at time $t$ to label the overlapping particles). The first term in $g_{4}(r, t)$ is a pair correlation function restricted to the subset of overlapping particles. The second term represents the probability of any two randomly chosen particles overlapping at times 0 and $t$. The structure factor $S_{4}(\boldsymbol{q}, t)$ that corresponds to $g_{4}(\boldsymbol{r}, t)$ is its Fourier transform

$$
S_{4}(\boldsymbol{q}, t)=\int g_{4}(\boldsymbol{r}, t) \exp [-\mathrm{i} \boldsymbol{q} \cdot \boldsymbol{r}] \mathrm{d} \boldsymbol{r} .
$$

Equation (8) implies that

$$
\lim _{\boldsymbol{q} \rightarrow 0} S_{4}(\boldsymbol{q}, t)=\frac{\chi_{4}(t)}{\beta} .
$$

Equation (8) is analogous to the static structure factor $S(\boldsymbol{q})$, but 'scatters' off overlapping particles using information on overlapping particles at time $t$ to label particles at time 0 .

\subsection{Self and distinct contributions to $Q(t), \chi_{4}(t), g_{4}(r, t)$ and $S_{4}(q, t)$}

The contribution of a given particle $i$ to $Q(t)$ is a result of three possible events: (i) particle $i$ remains within a distance $a$ of its original position; (ii) particle $i$ moves and is replaced (within a distance $a$ ) by another particle; or (iii) particle $i$ moves a distance greater than $a$ and is not replaced by another particle. Case (iii) does not count as an overlap, and thus does not contribute to $Q(t)$. Cases (i) and (ii) count as overlaps and contribute to the value of $Q(t)$. Cases (ii) and (iii) belong to the set of delocalized particles. However, cases (i) and (ii) clearly represent two very different physical situations. To elucidate the various contributions to the four-point correlation function, we separate $Q$ into self and distinct components,

$Q(t)=Q_{\mathrm{S}}(t)+Q_{\mathrm{D}}(t)=\sum_{i=1}^{N} w\left(\left|\boldsymbol{r}_{i}(t)-\boldsymbol{r}_{i}(0)\right|\right)+\sum_{i=1}^{N} \sum_{i \neq j}^{N} w\left(\left|\boldsymbol{r}_{i}(0)-\boldsymbol{r}_{j}(t)\right|\right)$.

The self part, $Q_{\mathrm{S}}(t)$, measures the number of particles that move less than a distance $a$ in a time interval $t$; we call these 'localized' particles. The distinct part, $Q_{\mathrm{D}}(t)$, measures the number of particles replaced within a radius $a$ by another particle in time $t$; we call these 'replaced' particles.

Following the scheme of decomposing $Q(t), \chi_{4}(t)$ can be decomposed into self $\chi_{\mathrm{SS}}(t)$, distinct $\chi_{\mathrm{DD}}(t)$, and cross $\chi_{\mathrm{SD}}(t)$ terms: $\chi_{4}(t)=\chi_{\mathrm{SS}}(t)+\chi_{\mathrm{DD}}(t)+\chi_{\mathrm{SD}}(t)$, where $\chi_{\mathrm{SS}}(t) \propto\left\langle Q_{\mathrm{S}}^{2}(t)\right\rangle-\left\langle Q_{\mathrm{S}}(t)\right\rangle^{2}, \chi_{\mathrm{DD}}(t) \propto\left\langle Q_{\mathrm{D}}^{2}(t)\right\rangle-\left\langle Q_{\mathrm{D}}(t)\right\rangle^{2}$ and $\chi_{\mathrm{SD}}(t) \propto\left\langle Q_{\mathrm{S}}(t) Q_{\mathrm{D}}(t)\right\rangle-$ $\left\langle Q_{\mathrm{S}}(t)\right\rangle\left\langle Q_{\mathrm{D}}(t)\right\rangle$. Thus $\chi_{\mathrm{SS}}(t)$ is the susceptibility arising from fluctuations in the number of localized particles, $\chi_{\mathrm{DD}}(t)$ is the susceptibility arising from fluctuations in the number of particles that are replaced by a neighbouring particle, and $\chi_{\mathrm{SD}}(t)$ represents cross fluctuations between the number of localized and replaced particles.

We also consider 'delocalized' particles, that is particles that in a time $t$ are more than a distance $a$ from their original location. As was pointed out in [2], substituting $1-w$ for $w$ in equation (2) gives the delocalized order parameter $Q_{\mathrm{DL}}(t)=N-Q_{\mathrm{S}}(t)$, and as a result, $\chi_{\mathrm{DL}}(t) \equiv \chi_{\mathrm{SS}}(t)$. 

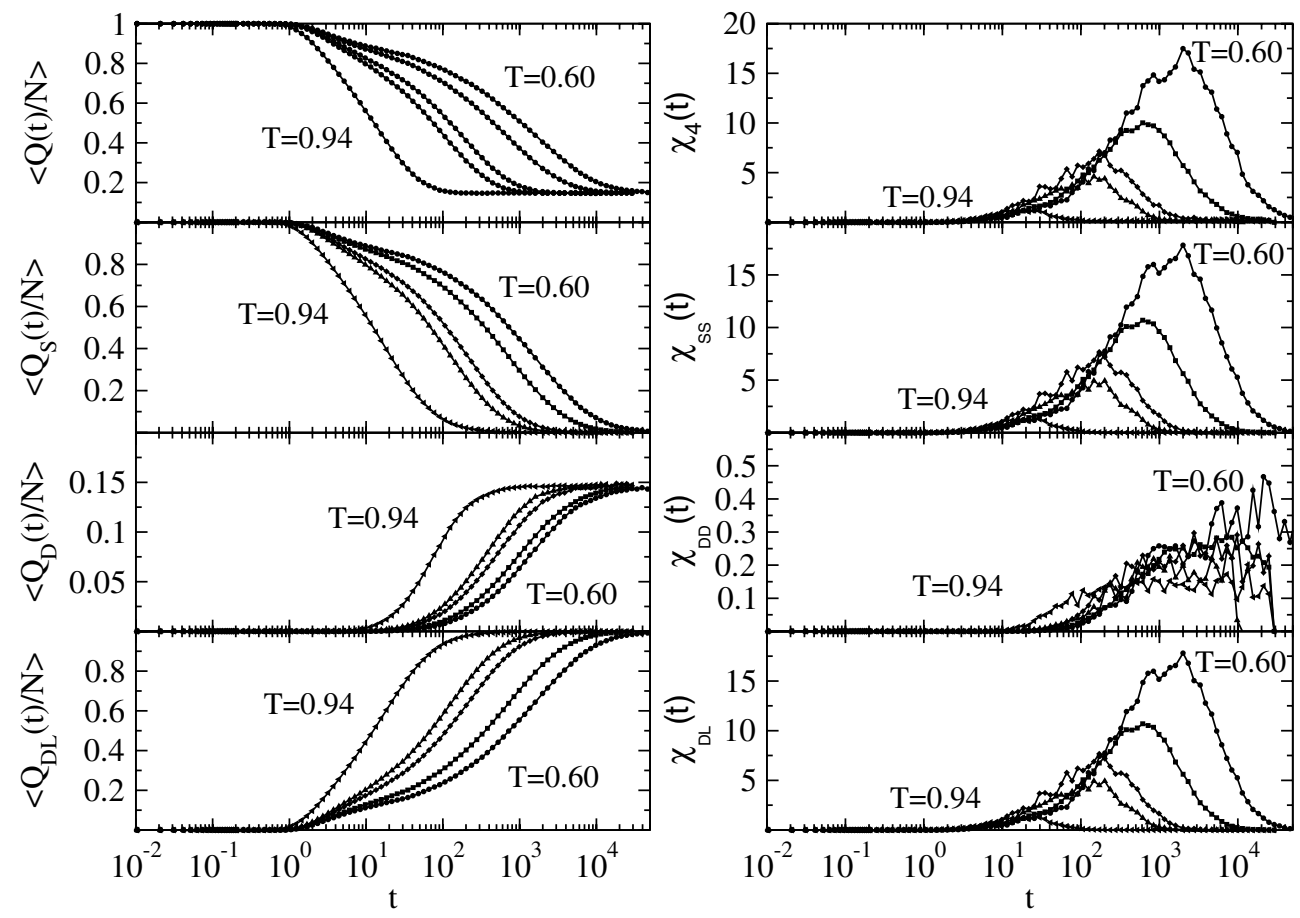

Figure 1. Temperature and time dependence of $\langle Q(t) / N\rangle,\left\langle Q_{\mathrm{S}}(t) / N\right\rangle,\left\langle Q_{\mathrm{D}}(t) / N\right\rangle,\left\langle Q_{\mathrm{DL}}(t) / N\right\rangle$, $\chi_{4}(t), \chi_{\mathrm{SS}}(t), \chi_{\mathrm{DD}}(t)$ and $\chi_{\mathrm{DL}}(t) \equiv \chi_{\mathrm{SS}}(t)$.

Likewise, we can find terms in $g_{4}(r, t)$ and $S_{4}(q, t)$ that correspond to localized, replaced and delocalized particles, e.g. $g_{4}(r, t)$ and $S_{4}(q, t)$ of localized particles correspond to $g_{4}^{\mathrm{SS}}(r, t)$ and $S_{4}^{\mathrm{SS}}(q, t)$ and for $i=j$ and $l=k$ in equations (7) and (8), respectively. In the next section we present numerical results for $S_{4}^{\mathrm{SS}}(q, t)$ and the corresponding correlation length $\xi_{4}^{\mathrm{SS}}(t)$.

\section{Results}

Figure 1 shows the time and temperature dependence of $Q(t)$ and $\chi_{4}(t)$ and their terms described in section 3. The figure shows that for all sufficiently low $T,\langle Q(t) / N\rangle$ and $\left\langle Q_{\mathrm{S}}(t) / N\right\rangle$ are characterized by a two-step relaxation, commonly observed in the intermediate scattering function [26], as a result of the transient caging of particles. $\left\langle Q_{\mathrm{DL}}(t) / N\right\rangle$ has the opposite time dependence from $\left\langle Q_{\mathrm{S}}(t) / N\right\rangle$ due to the fact that it measures the number of particles that moved a distance greater than $a$. The same applies to $\left\langle Q_{\mathrm{D}}(t) / N\right\rangle$ since those particles constitute the subset of delocalized particles. At short times, particles oscillate in a region smaller than the overlap radius $a$, and so $\langle Q(t) / N\rangle=1$ and $\left\langle Q_{\mathrm{S}}(t) / N\right\rangle=1$. We observe a short, initial relaxation of $Q(t)$ and $Q_{\mathrm{S}}(t)$, and a longer, secondary relaxation. $\chi_{4}(t)$, $\chi_{\mathrm{SS}}(t)$ and $\chi_{\mathrm{DL}}(t)$ are zero at short time, attain a maximum at some intermediate time $t_{4}^{\max }$, and decay at long time to zero in the thermodynamic limit.

$\chi_{4}(t)$ and its terms measure the correlated motion between pairs of particles, calculated equivalently from fluctuations in the number of localized, replaced and delocalized particles or from the corresponding four-point correlation functions. The behaviour of $\chi_{4}(t)$ demonstrates that correlations are time dependent, with a maximum at a time $t_{4}^{\max }$. Similar behaviour was 


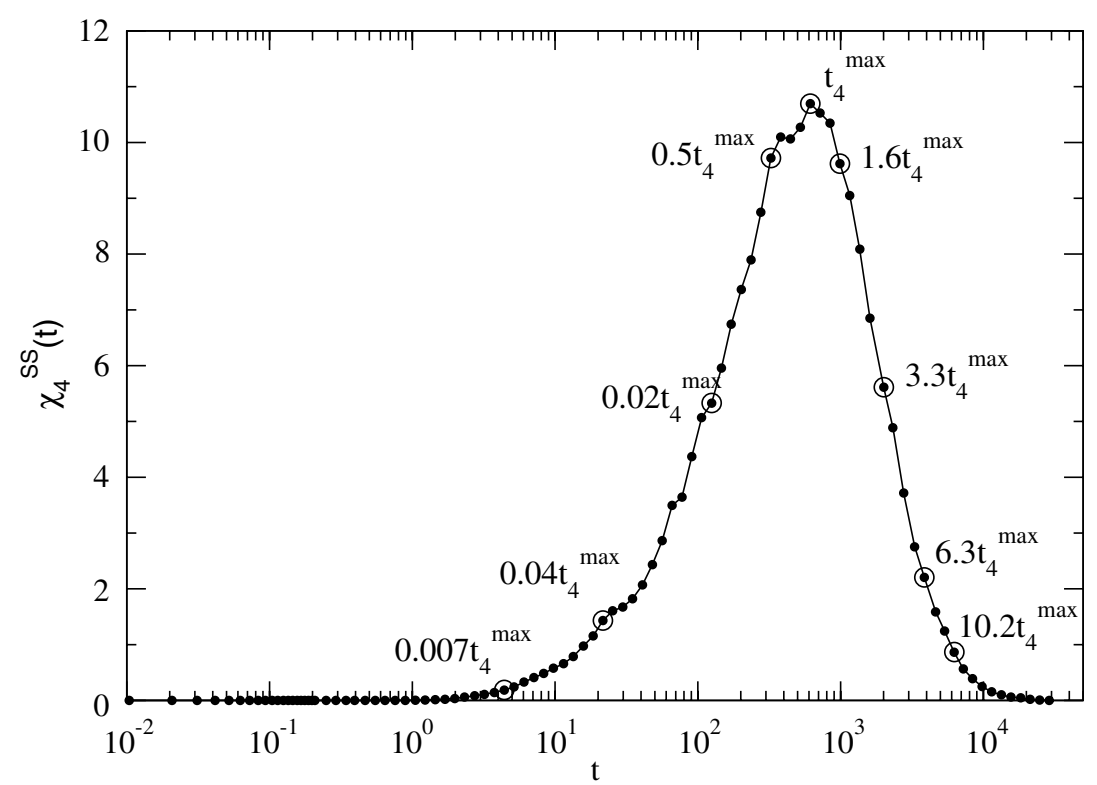

Figure 2. Time dependence of $\chi_{4}^{\mathrm{SS}}(t)$ at $T=0.62$. Times that are encircled and labelled with an appropriate fraction of $t_{4}^{\max }$ correspond to the snapshots in figure 3 .

reported for the same and other model liquids in [2, 42, 43] for a generalized susceptibility related to a displacement-displacement correlation function $\chi_{\mathrm{U}}(t)$, which measures the correlations between displacements of particles as a function of time. In these works, SHD was observed to be most pronounced in the late $\beta$ /early $\alpha$-relaxation regime. We find that the correlations measured by $\chi_{4}(t)$ are most pronounced in the $\alpha$-relaxation regime (see e.g. [4]).

Figure 2 shows the time dependence of $\chi_{\mathrm{SS}}(t)$ at $T=0.62$. There are nine points marked as open circles that correspond to the times at which we show localized and delocalized particles in figure 3.

The four-point structure factor of localized particles $S_{4}^{\mathrm{SS}}(q, t)$ calculated from equation (8) is plotted versus $q$ in figure 4 , at $T=0.60$. We also find that at very early times (when $\langle Q(t) / N\rangle=1), S_{4}^{\mathrm{SS}}(q, t)=S(q)$. We find that while $S(q)$ shows no change at small $q$ (see e.g. the static structure factor in [3]), $S_{4}^{\mathrm{SS}}(q, t)$ develops a peak at small $q$ which grows (figure 4(a)) and decays in time (figure 4(b)), indicating the presence of long-range correlations in the locations of overlapping particles.

Inspired by the Ornstein-Zernike theory (OZT) [44], which describes, for example, density fluctuations near a liquid-gas transition, we use the following function

$$
S_{4}^{\mathrm{SS}}(q, t)=\frac{S_{4}^{\mathrm{SS}}(0, t)}{\left[1+\left(q \xi_{4}^{\mathrm{SS}}(t)\right)^{2}\right]},
$$

where $S_{4}^{\mathrm{SS}}(0, t)$ and $\xi_{4}^{\mathrm{SS}}(t)$ are fitting parameters. The fitting was performed using an interiorreflective Newton method in MATLAB, and setting the termination tolerance of the function value to 0.1 . We find a good fit to the data in the $q$ range from $q=0.34$ to 1.9 , for each $T$ and time. The observed narrowing of the peak directly reveals the growing range of $g_{4}^{\mathrm{SS}}(r, t)$ with decreasing $T$.

The time and temperature dependence of $\xi_{4}^{\mathrm{SS}}(t)$ obtained from this fit is plotted for several state points in figure 5. We see that the qualitative behaviour of $\xi_{4}^{\mathrm{SS}}(t)$ is similar to that of 

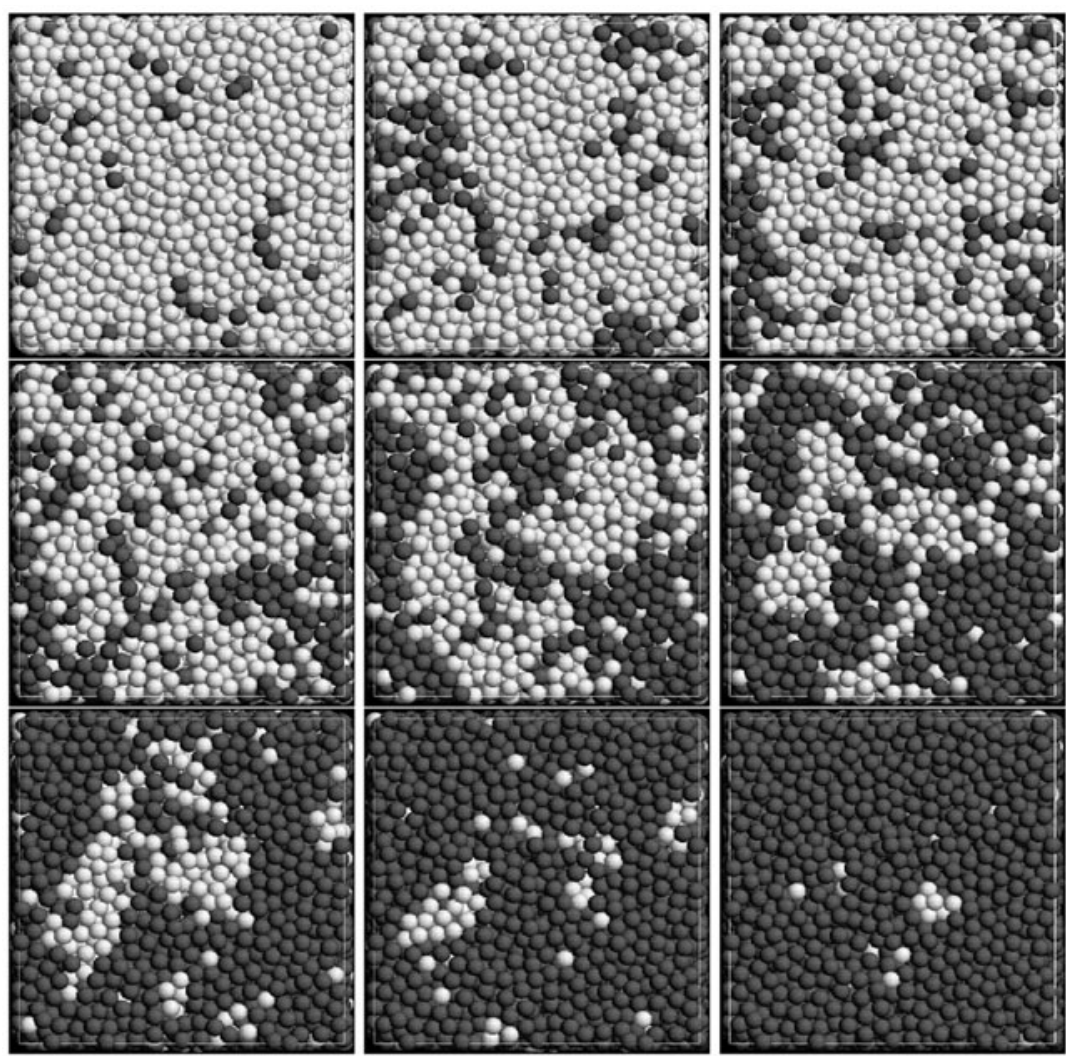

Figure 3. Localized (light grey) and delocalized (dark grey) particles at times $0.007 t_{4}^{\max }, 0.04 t_{4}^{\max }$, $0.2 t_{4}^{\max }, 0.5 t_{4}^{\max }, t_{4}^{\max }, 1.6 t_{4}^{\max }, 3.3 t_{4}^{\max }, 6.3 t_{4}^{\max }$ and $10.2 t_{4}^{\max }$.

$\chi_{4}^{\mathrm{SS}}(t): \xi_{4}^{\mathrm{SS}}(t)$ has a maximum in time that coincides with the maximum in $\chi_{4}^{\mathrm{SS}}(t)$, and as $T$ decreases, the amplitude and time of this maximum increase. The highest values of $\xi_{4}^{\mathrm{SS}}(t)$ for $T=0.60$ exceed half the simulation box size. The fit at these points depends strongly on the number of points used, initial parameter guesses and other details, and can yield large values (e.g. $>40$ ) depending on these details. Since these values greatly exceed the range over which we can meaningfully interpret the resulting correlation length, we make no attempt to define rigorously the upper error bounds at these points, but the data are well bounded from below. The fits at all other points and temperatures are well constrained. The length scale $\xi_{4}^{\mathrm{SS}}(t)$ characterizes the typical distance over which localized particles are spatially correlated.

\section{Discussion}

In this paper, we have focused on a four-point, time-dependent density correlation function $g_{4}^{\mathrm{SS}}(r, t)$ and corresponding time-dependent structure factor $S_{4}^{\mathrm{SS}}(q, t)$, and demonstrated that these functions are sensitive to dynamical heterogeneity in a model glass-forming liquid. As derived in previous works $[1-3,45,46]$, this correlation function is related to an order parameter $Q(t)$ corresponding to the number of 'overlapping' particles in a time window $t$, where the term 'overlap' is used to denote a particle that was either localized or replaced in a time $t$.

We calculated the correlation length of localized particles $\xi_{4}^{\mathrm{SS}}(t)$, characterizing the range of $g_{4}^{\text {SS }}(r, t)$, and showed that it depends on time and attains its maximum value in the 


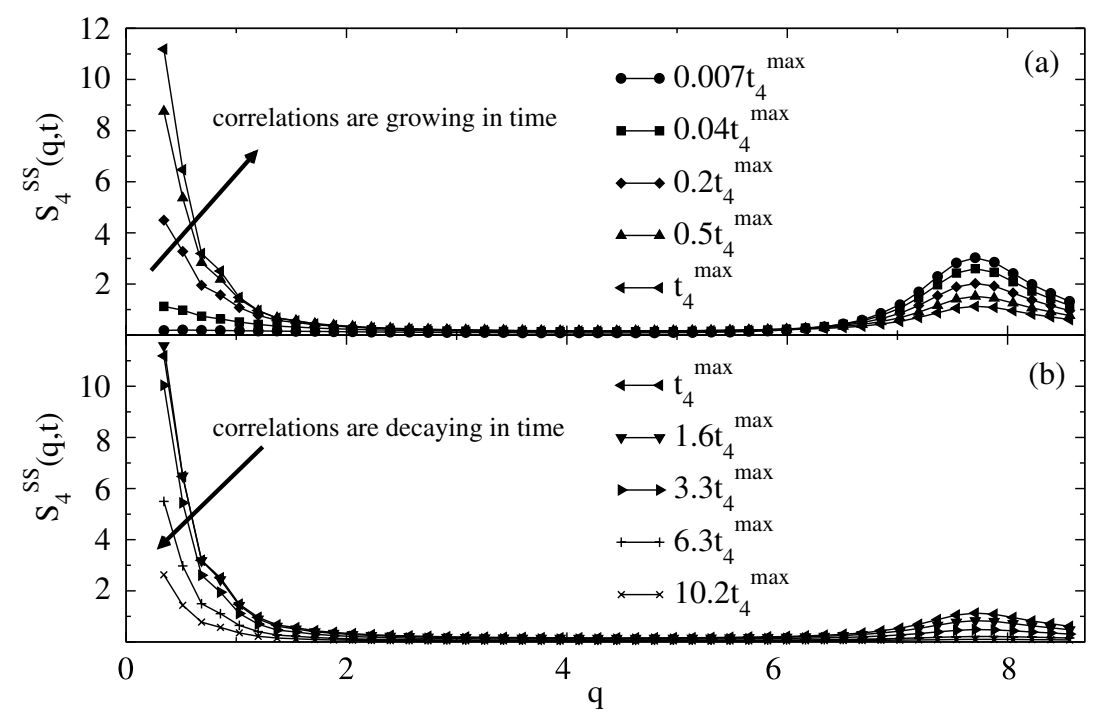

Figure 4. Time dependence of $S_{4}^{S S}(q, t)$ at $T=0.62 . S_{4}^{S S}(q, t)$ is shown at times identical to those shown for $g_{4}(r, t)$ in figure 2. Note that the height of the first diffraction peak in $S_{4}^{\text {SS }}(q, t)$ decreases monotonically as a function of time. This is because it depends on the number of localized particles, which also decreases monotonically in time (see figure 1).

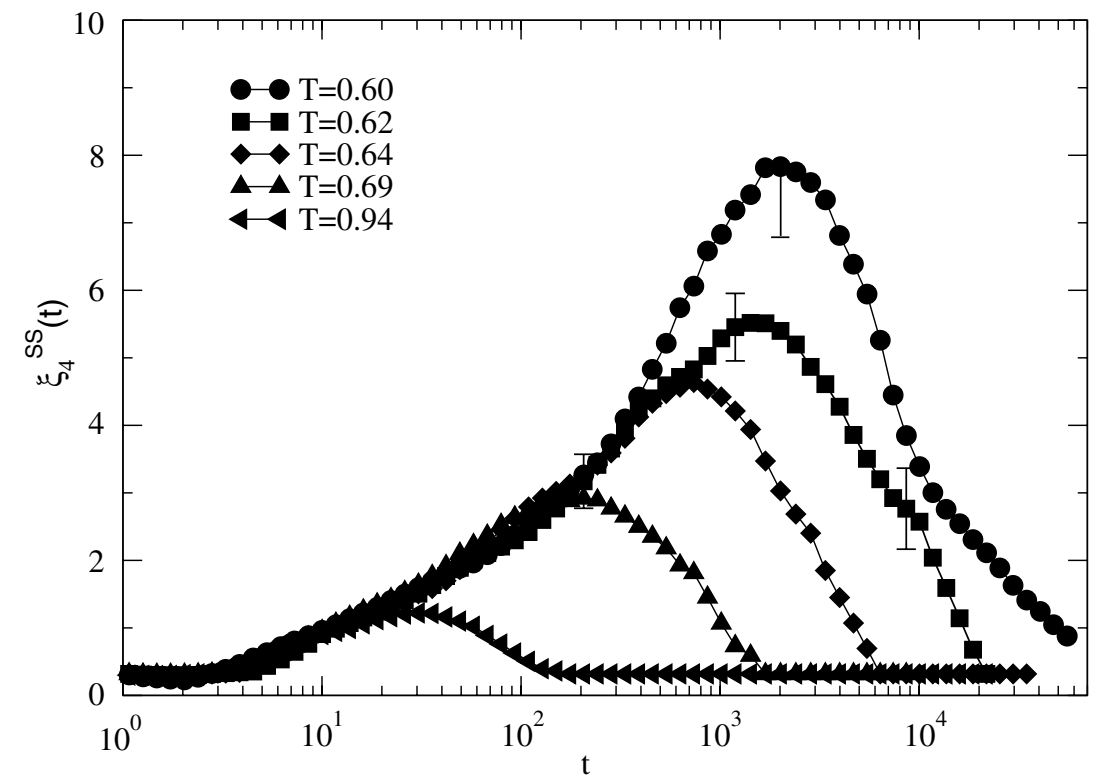

Figure 5. Time and temperature dependence of $\xi_{4}^{\mathrm{SS}}(t)$ obtained from the fits to equation (11). The data shown are smoothed over successive groups of five points.

$\alpha$-relaxation regime. We also showed that this maximum grows to exceed half of the simulation box size, close to $T_{\mathrm{MCT}}$. This length scale characterizes the typical size of dynamically localized domains. 
The characteristic length scale calculated here is related to length scales calculated from the displacement-displacement correlation function [43], cluster size [47] and other measures of correlated particle motion and dynamical heterogeneity [48-50]. $\xi_{4}^{\mathrm{SS}}(t)$ is essentially the same as that obtained by considering the delocalized particles (the set of particles that in any time window $t$ moves beyond a distance $a$ ) due to the mathematical identity between $\chi_{4}$ for localized and delocalized particles. This suggests a picture of fluctuating domains of temporarily localized and delocalized particles, perhaps similar to that proposed by Stillinger and Hodgdon [24].

Finally, we note that all quantities presented here can be measured in dense colloidal suspensions using confocal microscopy studies [51, 52].

\section{References}

[1] Franz S, Donati C, Parisi G and Glotzer S C 1999 Phil. Mag. B 791827 Donati C, Franz S, Glotzer S C and Parisi G 2002 J. Non-Cryst. Solids 307215

[2] Glotzer S C, Novikov V N and Schrøder T B 2000 J. Chem. Phys. 112509

[3] Lačević N et al 2002 Phys. Rev. E 66030101

[4] Lačević N, Starr F W, Schrøder T B and Glotzer S C 2003 Preprint

[5] Lačević N 2003 Dissertation The Johns Hopkins University

[6] Weintraub H et al 1995 Science 2671609

[7] Debenenedetti P G 1996 Metastable Liquids: Concepts and Principles (Princeton, NJ: Princeton University Press)

[8] Harrison G 1976 Dynamics Properties of Supercooled Liquids (London: Academic)

[9] Knight C 1967 The Freezing of Supercooled Liquids (Princeton, NJ: Van Nostrand Reinhold) (Published for the Commission on College Physics by Van Nostrand)

[10] Fourkas J T (ed) Supercooled Liquids: Advances and Novel Applications (ACS Symp. Ser. 0097-6156) (Washington, DC: American Chemical Society) p 676

[11] Glotzer S C (ed) 1995 Comput. Mater. Sci. 4

[12] Franz S, Glotzer S C and Sastry S (ed) 2000 ICTP-NIS Conf. on Unifying Concepts in Glass Physics; J. Phys.: Condens. Matter 12 (special issue)

[13] Giordano M, Leporini D and Tosi M P (ed) 1996 Non Equilibrium Phenomena in Supercooled Fluids, Glasses and Amorphous Materials (Singapore: World Scientific)

[14] Strandburg K J (ed) 1992 Bond-Orientational Order in Condensed Matter Systems (New York: Springer) (foreword by D R Nelson)

[15] Liu A J and Nagel S R (ed) 2001 Jamming and Rheology: Constrained Dynamics on Microscopic and Macroscopic Scales (New York: Taylor and Francis)

[16] Ferry D J 1980 Viscoelastic Properties of Polymers (New York: Wiley)

[17] Dixon P K et al 1990 Phys. Rev. Lett. 651108

[18] Tolle A et al 1997 Phys. Rev. E 56809

[19] Frick B, Richter D and Ritter C 1989 Europhys. Lett. 9557

[20] Kartini E et al 1996 Phys. Rev. B 546292

[21] Leheny R L et al 1996 J. Chem. Phys. 1057783

[22] Deppe D D, Miller R D and Torkelson J M 1996 J. Polym. Sci. B 342987

[23] Hall D B, Dhinojwala A and Torkelson J M 1997 Phys. Rev. Lett. 79103

[24] Hodgdon J A and Stillinger F H 1993 Phys. Rev. E 48207

[25] Stillinger F H and Hodgdon J A 1994 Phys. Rev. E 502064

[26] Sillescu H 1999 J. Non-Cryst. Solids 24381

[27] Glotzer S C 2000 J. Non-Cryst. Solids 274342

[28] Ediger M D 2000 Annu. Rev. Phys. Chem. 5199

[29] Bohmer R 1998 Curr. Opin. Solid State Mater. Sci. 3378

[30] Richert R 2002 J. Phys.: Condens. Matter 14 R703

[31] Bohmer R et al 1998 J. Non-Cryst. Solids 2351

[32] Garrahan J P and Chandler D 2002 Phys. Rev. Lett. 8903570

[33] Wahnstrom G 1991 Phys. Rev. A 443752

[34] Schrøder T B 2000 Preprint cond-mat/0005127

[35] Schrøder T B et al 2000 J. Chem. Phys. 1129834 
[36] Schrøder T B and Dyre J C 1998 J. Non-Cryst. Solids 235331

[37] Novikov V N and Sokolov A P 2003 Phys. Rev. E 67031507

[38] Franz S and Parisi G 2000 J. Phys.: Condens. Matter 126335

[39] Kirkpatrick T R and Wolynes P G 1987 Phys. Rev. A 353072

Kirkpatrick T R and Wolynes P G 1987 Phys. Rev. A 36852

Kirkpatrick T R and Thirumalai D 1987 Phys. Rev. B 365388

Kirkpatrick T R and Thirumalai D 1995 Transp. Theory Stat. Phys. 24927

[40] Crisanti A, Horner H and Sommers H J 1993 Z. Phys. B 92257

[41] Barrat A 1997 Preprint cond-mat/9701031

[42] Bennemann C, Donati C, Baschnagel J and Glotzer S C 1999 Nature 399246

[43] Glotzer S C, Donati C and Poole P H 1999 Computer Simulation Studies in Condensed-Matter Physics XI (Springer Proc. Phys. vol 84) ed D P Landa and H-B Schüttler (Berlin: Springer) p 212

Donati C, Glotzer S C and Poole P H 1999 Phys. Rev. Lett. 825064

[44] Stanley H E 1971 Introduction to Phase Transitions and Critical Phenomena (New York: Oxford University Press)

[45] Dasgupta C et al 1991 Europhys. Lett. 15467

[46] Franz S and Parisi G 1998 Preprint cond-mat/9804084

[47] Gebremichael Y et al 2001 Phys. Rev. E 64051503

[48] Doliwa B and Heuer A 2000 Phys. Rev. E 616898

[49] Yamamoto R and Onuki A 1998 Phys. Rev. E 583515

[50] Melcuk A I et al 1995 Phys. Rev. Lett. 752522

[51] Weeks E R et al 2000 Science 287627

[52] Kegel W K and van Blaaderen A 2000 Science 287290 